\title{
Digital rectal examination in prostate cancer screening
}

\author{
Angela Zhang (Meds 2015), Thomas Fear (Meds 2015), and Hammood Ahmed (Meds 2015) \\ Faculty Reviewer: Dr. John Jordan, MD (Department of Family Medicine)
}

\section{INTRODUCTION}

Digital rectal examination (DRE) may be performed in order to identify rectal disease, prostate cancer, examine anal sphincter tone, or to examine female gynecological structures. Rectal examination has been described by conflicting opinions as both a cause of "more harm than good" and conversely as a "crucial part of the examination [of a patient]".,1,2 Despite its many diagnostic applications, the test is also uncomfortable for some patients, doctors, and medical students. This article will focus on the characteristics of the test in prostate cancer screening, and how the challenges of patient resistance and medical student education can be addressed.

\section{ROLE OF DRE IN PROSTATE CANCER SCREENING}

What lies at the center of the DRE debate is its utility for identifying and diagnosing different diseases. One application for DRE is for the detection of prostate cancer which is the most common cancer in men other non-melanoma skin cancers. It was diagnosed in 26,500 Canadians in 2012, and resulted in 4000 deaths. ${ }^{3}$ However the disease is often indolent, and while one in seven men are diagnosed, only one in 27 men die from the disease. ${ }^{4}$ The value of DRE can come into question because studies conflict as to whether screening for prostate cancer can lower morbidity. ${ }^{4}$ Nonetheless, because of the frequency of the disease and the possibility detecting the disease when it is curable many patients and physicians choose to screen.

The DRE is an inexpensive test that involves assessment of prostate size, consistency, mobility, and irregularities in shape. However the size alone is not a useful predictor of prostate cancer risk and there is low correlation between transrectal ultrasonography (TRUS) measured prostate volume and DRE estimated volume. ${ }^{5,6}$ Additionally, while carcinomas are felts as hard irregular nodules similar indurations may also be caused by benign prostatic hypertrophy (BPH) or calculi. This means an examiner must order further tests when any induration is felt, and repeat the test regularly to detect for any changes or progression. ${ }^{5,4}$ The exam also requires technical skill. Not all examiners can palpate the entire posterior surface of the prostate. The ability of an examiner to reach and examine the apex of the prostate, half of the prostate, three quarters of it, and the entire prostate in a recent study was $93.7 \%, 66.3 \%, 23.2 \%$ and $3.2 \%$ for these respective components of the prostate gland. ${ }^{7}$ Evidence for the importance of technical skill in DRE also comes from studies of inter-examiner variation. One study compared consultant and resident urologists who performed DREs to determine if prostates were suspicious for cancer. The overall agreement was expressed with a kappa statistic of 0.22 , which suggests only fair agreement between examiners. However, while the kappa statistic among residents was 0.25 ; among consultants it was 0.63 which suggests a substantial level of agreement. ${ }^{8}$ Further research has shown that more experienced urologists tend to as- sess the size of a prostate more accurately. ${ }^{9}$ These findings support the idea that technical skill is important to successfully performing DREs. One means to address this is through improved education of medical trainees, a topic addressed later in this article.

When DRE is used to screen for prostate cancer in men over 50 years of age, the cancer detection rate is $3.2 \%$ with a positive predictive value of $21 \%$. The sensitivity and specificity of DRE are $21 \%$ and $86 \%$ respectively. ${ }^{10}$ It is also recommended that DRE is performed in conjunction with prostate specific antigen (PSA) in prostate cancer screening because this improves the overall sensitivity. Whereas the prostate cancer detection rate was $3.2 \%$ for digital rectal examination, it was $4.6 \%$ for PSA and 5.8\% for the 2 methods combined. ${ }^{11}$ Of note is that the detection rates of the two tests only partially overlap, and together the two tests detect more cases of prostate cancer.

In conclusion, because the disease is relatively indolent, it is recommended that an informed decision is made by patients whether or not to screen for prostate cancer. Because the test is technically difficult to perform, steps should be taken to improve the education of medical trainees. If screening is to be performed, then regular DRE is recommended to be used conjunction with PSA testing to increases the rate of cancer detection.

\section{PATIENT BARRIERS}

Some patient barriers to the acceptance of DREs include discomfort, embarrassment and men associating the exam with homosexuality. Furthermore some men are worried about feeling stimulated and having an unwanted and uncontrolled physical response during a DRE. Men may not seek medical attention that includes DRE until they're no longer able to cope with symptoms; this could be due to both fear of the procedure or fear of discovering illness. One way to improve patient acceptance of DRE is to improve patient education. Ample information on the process and reason for performing the procedure should be provided for patients. To be included in the conversation could be discussion about past experiences with DRE, whether positive or negative. Positioning can also be chosen to reduce patient embarrassment during the procedure. The left lateral position has been show to be the least embarrassing without compromising the results of the test. ${ }^{12}$ Furthermore, many patients view colonoscopy drastically differently than DRE. The reason is that colonoscopy is often perceived as more technological and hence perceived as more scientific. Therefore extra care should be taken by physicians to maintain a professional environment during DRE. Language used should be chosen carefully. For example, phrases like 'examine' the prostate rather than 'feel' the prostate make the process sound more clinical and scientific, hence more accepted by patients who are wary of the procedure. ${ }^{13}$

\section{TEACHING OF THE DRE}


DIAGNOSTIC REVIEW

One factor limiting the usefulness of DRE is inter-examiner variability, which results in inconsistent exam findings. This has promoted studies that examine how the digital rectal exam is taught to medical students and residents. Teaching during medical school has a huge bearing on a physician's future skill in performing DRE, yet several aspects limit what students learn. DRE is often learned through textbook readings and facilitator demonstrations. The three dimensional nature of the prostate is difficult to convey in textbooks. In demonstrations, it is challenging to teach the subtleties of palpation because DRE is a very internal procedure. ${ }^{14}$

One attempt to solve this problem uses rectal simulators which are currently being developed to teach the DRE. A mannequin modeling the rectum and prostate is attached to electronic sensors, so that when an examiner palpates the rectum sensors analyze the pressure and location of palpation for instructors to assess and give feedback. Beyond use as a teaching tool, this simulator can also detect differences in performance between examiners and so attempts to quantify inter-examiner variability in DRE.

University of California Medical School developed a DRE teaching model that include didactic lectures, practical learning by performing the procedure on a standardized patient, and small group sessions led by faculty members. In a study conducted by Kaplan et. al, students' comfort level in terms of performing DRE were comparing between group of students that received DRE training from the new teaching model and students that didn't receive formal training in performing DRE. Students who had received the teaching model were more comfortable performing DREs. Examiner comfort and confidence level is crucial to patient comfort and confidence; hence this model of learning through three modalities should be given consideration for more widespread use in the teaching of medical students. ${ }^{15}$

\section{CONCLUSION}

Despite differences in prostate cancer screening guidelines between various countries, the majority of countries recommend a combination approach of serum PSA with DRE. ${ }^{16}$ Therefore DRE is still an important part of undergraduate and postgraduate medical curricula.

Things to keep in mind:

- $\quad$ Reassure your patient: explain to them why you're doing this exam and what to expect during the exam.

- Use medical terms to describe procedure: examine, palpate, drape, examination table etc.

- $\quad$ Ask the patient if they have ever had a bad experience during a DRE.

- Position choice: left lateral position is perceived by patients to be least embarrassing, while achieving similar results as other positions. ${ }^{16}$

There are limitations to use of DRE in prostate cancer screening. However as a procedure that is low risk, fast and office setting procedure when used in combination with PSA increase cancer detection comparing to PSA alone, there is still a place for DRE in medicine and prostate cancer screening today. There is inter-examiner variability meaning that experience level has an impact on efficacy of this test. Therefore DRE should to be more rigorously taught to new generations of physicians and care should be taking regarding how DRE is taught. Modifications should be made to the teaching of this procedure in medical schools. Traditional learning from texts and demonstrations should be enhanced by the incorporation of modern technology like DRE simulators and more comprehensive teaching models to educate future physicians.

\section{REFERENCES}

1. Spence D. Bad Medicine: Digital Rectal Examination. Brit Med J. 2011 Jun; $1 ; 342: \mathrm{d} 3421$.

2. Snape J, Elliott B. Good Medical Practice. Brit Med J. 2001 Jun;28;342:d3949.

3. Prostate cancer statistics at a glance [Internet]. Toronto (ON): 2012 Canadian Cancer Society; 2005 Jan 1 [updated 2012 May 1; cited 2012 May 4]. Available from: http://www.cancer.ca/Ontario/About $\% 20$ cancer/Cancer\%20statistics/Stats\%20at\%20a\%20glance/Prostate\%20cancer.aspx?sc_lang=en\&r=1

4. Izawa, JI, Klotz J, Siemens RD, Kassouf W, So A, Jordan J, Chetner M, Iansavichene A. Prostate cancer screening: Canadian guidelines 2011. CUAJ-Can Urol Assoc. 2011 August;5(4):235-240.

5. Longo DL, Fauci AS, Kasper DL, Hauser SL, Jameson JL, Loscalzo J. Harrison's principles of internal medicine. 18th ed. New York: McGraw-Hill Medical Publishing Division; c2012. Chapter 95, Benign and Malignant Diseases of the Prostate; p. 796-805.

6. Roehrborn CG, Girman CJ, Rhodes T, Hanson KA, Collins GN, Sech SM, et al. Correlation between prostate size estimated by digital rectal examination and measured by transrectal ultrasound. Urology. 1997 Apr;49(4):548-57.

7. Koulikov D, Mamber A, Fridmans A, Abu Arafeh W, Shenfeld OZ. Why I cannot find the prostate? Behind the subjectivity of rectal exam. ISRN Urology. 2012 Feb;2012. Epub 2012 Feb 15. Mar 19:[4 p.].

8. Smith DS, Catalona WJ. Interexaminer variability of digital rectal examination in detecting prostate cancer. Urology. 1995 Jan;45(1):70-4.

9. Cheng WC, Ng FC, Chan KC, Cheung YH, Chan WL, Wong SW. Interobserver variation of prostatic volume estimation with digital rectal examination by urological staffs with different experiences. International Braz J Urol. 2004 Nov-Dec;30(6):466-71.

10. Richie JP, Catalona WJ, Ahmann FR, Hudson MA, Scardino PT, et al. Effect of patient age on early detection of prostate cancer with serum prostate-specific antigen and digital rectal examination. Urology. 1993 Oct: 42(4):365-74.

11. Catalona WJ, Richie JP, Ahmann FR, Hudson MA, Scardino PT, Flanigan $\mathrm{RC}$, et al. Comparison of digital rectal examination and serum prostate specific antigen in the early detection of prostate cancer: results of a multicenter clinical trial of 6,630 men. J Urology. 1994 May;151(5):1283-90.

12. Romero FR, Romero AW, Tambara Filho R, Brenny Filho T, de Oliveira Junior FC. Patient positioning during digital rectal examination of the prostate: preferences, tolerability, and results. International Braz J Urol. 2011 MayJun;37(3):371-7.

13. Winterich JA, Quandt SA, Grzywacz JG, Clark PE, Miller DP, Acuna J, et al. Masculinity and the body: how African American and White men experience cancer screening exams involving the rectum. Am J Mens Health. 2009 Dec;3(4):300-9.

14. Balkissoon R, Blossfield K, Salud L, Ford D, Pugh C. Lost in translation: unfolding medical students' misconceptions of how to perform a clinical digital rectal examination. Am J Surg. 2009 Apr;197(4):525-32.

15. Kaplan AG, Abdelshehid CS, Alipanah N, Zamansani T, Lee J, Kolla SB, et al. Genitourinary exam skills training curriculum for medical students: a followup study of comfort and skill utilization. J Endourol. 2012 Oct;26(10):1350-5.

16. Gomella LG, Liu XS, Trabulsi EJ, Kelly WK, Myers R, Showalter T, et al. Screening for prostate cancer: the current evidence and guidelines controversy. Can J Urol. 2011 Oct;18(5):5875-83. 